\title{
New Control Design for Bounded Backstepping under Input Delays*
}

\author{
Frédéric Mazenc $^{\mathrm{a}}$ and Michael Malisoff ${ }^{\mathrm{b}}$ \\ ${ }^{\text {a } E P I ~ D I S C O ~ I N R I A-S a c l a y, ~ t h e ~ L a b o r a t o i r e ~ d e s ~ S i g n a u x ~ e t ~ S y s t e ̀ m e s ~(L 2 S, ~ U M R ~ C N R S ~ 8506), ~ C N R S, ~ C e n t r a l e S u p e ́ l e c, ~}$ \\ Université Paris-Sud, 3 rue Joliot Curie, 91192, Gif-sur-Yvette, France. \\ ${ }^{\mathrm{b}}$ Department of Mathematics, Louisiana State University, Baton Rouge, LA 70803-4918 USA.
}

\begin{abstract}
We provide a new backstepping result for time-varying systems with input delays. The novelty of our work is in the bounds on the controls, and the facts that (i) one does not need to compute any Lie derivatives to apply our controls, (ii) the controls have no distributed terms, and (iii) we do not require any differentiability conditions on the available controls for the subsystems.
\end{abstract}

Key words: Backstepping, delay, stabilization.

\section{Introduction}

Backstepping is useful for designing feedback controls for many classes of nonlinear systems. The basic idea of backstepping is to design globally asymptotically stabilizing controllers, by building up from known globally asymptotically stabilizing controllers for certain subsystems of the original system, often by constructing Lyapunov functions for the closed loop subsystems. Many contributions have been written on this subject; see Karafyllis (2002); Khalil (2002); Malisoff and Mazenc (2009). However, only a few works (such as Choi and Lim (2010); Mazenc and Bliman (2006); Mazenc et al. (2011)) apply backstepping to input delayed systems and none of those works provide bounded feedbacks. In Mazenc and Bowong (2004), a bounded backstepping feedback for time-varying systems is designed, but we are not aware of any extension of Mazenc and Bowong (2004) in the literature to cases with input delays.

There are several valuable techniques for designing controls under input delays; see Bresch-Pietri et al. (2012); Kharitonov and Niculescu (2003); Niculescu (2001). One

\footnotetext{
* Corresponding author: F. Mazenc, Tel.: + 33 [0] 1698517 62, Mobile: 06070423 52, Fax: + 33 [0] 1698517 65. A preliminary version of some of this work (excluding Proposition 1 and Section 8) appeared in the proceedings of the $2015 \mathrm{Eu}-$ ropean Control Conference (ECC). For a comparison between our ECC article and this journal version, see the end of Section 1 below. Supported by l'Institut pour le Contrôle et la Décision de l'Idex Paris-Saclay (iCODE) (Mazenc) and NSF Division of ECCS Grants 1102348 (Malisoff) and 1408295 (Malisoff).

Email addresses:

frederic.mazenc@12s. centralesupelec.fr (Frédéric

Mazenc), malisoff@lsu.edu (Michael Malisoff).
}

involves prediction, where the dynamic controls have distributed terms that usually require values of the state along a continuum of past times (but see Karafyllis and Krstic (2014); Karafyllis et al. (2015) for sampling based prediction). Prediction has its origins in the Smith predictor for linear systems in Smith (1959). However, recent advances in prediction make stabilization possible for many classes of nonlinear systems of ordinary and partial differential equations, including adaptive cases. See, e.g., the recent monographs Krstic (2009); Krstic and Bekiaris-Liberis (2013) and Bekiaris-Liberis and Krstic (2013) for some state-ofthe-art work related to prediction. While prediction allows arbitrarily long delays, the distributed terms may not always be easy to compute.

An alternative approach involves (a) building a control that ensures global asymptotic stability when the delay is zero and then (b) introducing a delay $h>0$ into the control and computing upper bounds on the allowable delays $h$ such that the closed loop system with the input delay $h$ is still globally asymptotically stable. This approach is sometimes called emulation, and it usually does not allow arbitrarily long delays (but see Mazenc et al. (2008) for drift-free control affine systems where a scaling of the control can ensure uniform global asymptotic stability under arbitrarily long delays). For control affine systems, the nominal controller in step (a) is often computed using Lie derivatives of a Lyapunov function for the corresponding undelayed system.

In this note, we propose a new extension of backstepping for time-varying systems that produces bounded controllers under input delays, and that differs from both prediction and emulation. Three potential advantages of our new approach are that (1) the control formula does not involve Lie derivatives along any vector fields of the systems, (2) our 
new controllers do not have any distributed terms, and (3) we can allow cases where the nominal controller is not necessarily $C^{1}$. Moreover, we can often allow arbitrarily long input delays, and all trajectories of the closed loop system converge to 0 . Our main assumption is a stability condition on a closed loop dynamics that is obtained by putting a suitably averaged nominal control into a subsystem of the original system; see (3).

In the next section, we provide definitions, to help make our work self contained. In Section 3, we state our bounded backstepping theorem under input delays, which we prove in Section 4. In Section 5, we give Lyapunov-like sufficient conditions for the main assumption of our theorem to hold. Our sufficient conditions ask that the nominal control stabilizes the system when the delay is set to zero. We illustrate our theorem in two examples, in Sections 6-7. In our first example, no negative values of the first components of the state are available for measurement (so the control depends on time delayed values of the output $y=(\max \{0, x\}, z)$ instead of current values, where $(x, z)$ is the state of the system) but there is an upper bound on the allowable delays. In our second example, our method applies without imposing any upper bound on the delay, using a control scaling argument. In both examples, the assumptions of our theorem are satisfied, so we can certify stability for the cascade under time delays. In Section 8, we provide a technical discussion that compares our new backstepping theorem with Mazenc et al. (2008) and other related results. We close in Section 9 by summarizing our contribution and by suggesting future research topics.

This paper generalizes our conference version Mazenc and Malisoff (2015). Our work Mazenc and Malisoff (2015) was confined to the case where the $z$ subsystem in Theorem 1 below is scalar valued, and Mazenc and Malisoff (2015) did not include Section 8 below, nor did Mazenc and Malisoff (2015) include simulations or Proposition 1 below on ways to check Assumption 2. By covering more general systems and ways to check Assumption 2, this version adds considerable value relative to Mazenc and Malisoff (2015).

\section{Definitions and Notation}

Unless otherwise noted, the dimensions of our vectors are arbitrary. The Euclidean norm of vectors in $\mathbb{R}^{n}$, and the induced norm of matrices, are both denoted by $|\cdot|$. For each constant $r>0$, we use $\mathcal{B}_{p, r}$ to denote the closed radius $r$ ball in $\mathbb{R}^{p}$ centered at the origin. For each integer $k \geq 0$, we let $C^{k}$ denote the set of all continuous functions whose partial derivatives up through order $k$ are all continuous, when the domains and ranges are clear from the context, so $C^{0}$ is the set of all continuous functions. We always assume that the initial times $t_{0} \geq 0$ for all trajectories are $t_{0}=0$, but analogous results can be written for all $t_{0} \geq 0$. Given any constant $h>0$, we let $C_{\text {in }}$ denote the set of all continuous functions $\phi:[-h, 0] \rightarrow \mathbb{R}^{n}$, which we call the set of all initial functions. A function $G:[0, \infty) \times \mathbb{R}^{n} \rightarrow \mathbb{R}^{p}$ is called uniformly bounded with respect to the first variable provided that there is a nondecreasing continuous function $\alpha:[0, \infty) \rightarrow[0, \infty)$ such that $|G(t, x)| \leq \alpha(|x|)$ holds for all $t \geq 0$ and $x \in \mathbb{R}^{n}$. We call a function $G:[0, \infty) \times \mathbb{R}^{n} \rightarrow \mathbb{R}^{p}$ uniformly locally Lipschitz in the second variable provided that for each constant $K>0$, there is a constant $L_{K}>0$ such that $|G(t, x)-G(t, y)| \leq L_{K}|x-y|$ holds for all $t \geq 0$ and for all $x$ and $y$ in $\mathcal{B}_{n, K}$. For each constant $r>0$, we define the (standard) saturation $\sigma_{r}: \mathbb{R} \rightarrow \mathbb{R}$ by $\sigma_{r}(p)=p$ if $p \in[-r, r]$ and $\sigma_{r}(p)=r \operatorname{sign}(p)$ for all $p \in \mathbb{R} \backslash[-r, r]$. Then

$$
\sigma_{n, r}(x)=\left(\sigma_{r}\left(x_{1}\right), \sigma_{r}\left(x_{2}\right), \ldots, \sigma_{r}\left(x_{n}\right)\right)
$$

denotes the corresponding vector saturation for each $x \in$ $\mathbb{R}^{n}$. Let $\mathcal{K}_{\infty}$ be the set of all $C^{0}$ functions $\gamma:[0, \infty) \rightarrow$ $[0, \infty)$ such that $\gamma(0)=0, \gamma$ is strictly increasing, and $\lim _{s \rightarrow \infty} \gamma(s)=\infty$. For any subset $S$ of a Euclidean space such that $0 \in S$, a function $\alpha: S \rightarrow[0, \infty)$ is called positive definite provided $\alpha(0)=0$ and $\alpha(r)>0$ for all $r \in S \backslash\{0\}$. A function $V:[0, \infty) \times \mathbb{R}^{n} \rightarrow[0, \infty)$ is called uniformly proper and positive definite provided there exist class $\mathcal{K}_{\infty}$ functions $\underline{\alpha}$ and $\bar{\alpha}$ such that $\underline{\alpha}(|x|) \leq V(t, x) \leq \bar{\alpha}(|x|)$ hold for all $t \geq 0$ and $x \in \mathbb{R}^{n}$. For each function $x:[-h, \infty) \rightarrow$ $\mathbb{R}^{n}$ and each $t \geq 0$, we define $x_{t} \in C_{\text {in }}$ by $x_{t}(\theta)=x(t+\theta)$, so $x_{t}$ is the translation operator. Let $\mathcal{L}^{2}(p,[0, \infty))$ denote the set of all Lebesgue measurable functions $d:[0, \infty) \rightarrow \mathbb{R}^{p}$ such that $\int_{0}^{\infty}|d(t)|^{2} \mathrm{~d} t<\infty$.

\section{Statement of Main Result and Remarks}

We consider the nonlinear time-varying system

$$
\dot{x}(t)=f(t, x(t))+g(t, x(t), z(t)), \quad \dot{z}(t)=u(t-h)
$$

where $x$ and $z$ are valued in $\mathbb{R}^{n}$ and $\mathbb{R}^{p}$, respectively, $u$ is the input, and $h>0$ is a constant delay (but see Remark 1 for generalizations). The initial functions $\left(x_{0}, z_{0}\right) \in C_{\text {in }}$ are arbitrary. We assume:

Assumption 1 The functions $f$ and $g$ in (1) are uniformly locally Lipschitz in the state vectors $x$ and $z, f(t, 0)=0$ for all $t$, and $f$ and $g$ are uniformly bounded with respect to the first variable. Also, for each continuous bounded function $z_{a}: \mathbb{R} \rightarrow \mathbb{R}^{p}$, all solutions of

$$
\dot{x}(t)=f(t, x(t))+g\left(t, x(t), z_{a}(t)\right)
$$

are defined for all $t \geq 0$.

Assumption 2 There are positive constants $q, \tau$, and $\bar{u}$ and a function $u_{\text {nom }}:[0, \infty) \times \mathbb{R}^{n} \rightarrow \mathcal{B}_{p, \bar{u}}$ such that $u_{\mathrm{nom}}$ is uniformly locally Lipschitz in the second variable and continuous, $u_{\text {nom }}(t, 0)=0$ for all $t \geq 0, q \tau<1$, and for each continuous function $d:[0, \infty) \rightarrow \mathbb{R}^{p}$ that exponentially converges to 0 , all solutions of

$$
\begin{aligned}
& \dot{\xi}(t)=f(t, \xi(t))+ \\
& g\left(t, \xi(t), \int_{t-\tau-h}^{t-h} \frac{q e^{q(\ell-t+h)}}{1-e^{-q \tau}} u_{\mathrm{nom}}(\ell, \xi(\ell)) \mathrm{d} \ell+d(t)\right)
\end{aligned}
$$

converge to 0 as $t \rightarrow \infty$.

Notice that we do not require $u_{\text {nom }}$ to be $C^{1}$. See Section 5 for ways to check that Assumption 2 is satisfied. In terms of the nominal control $u_{\text {nom }}$ from Assumption 2, we now 
consider the augmented system

$$
\left\{\begin{aligned}
\dot{x}(t)= & f(t, x(t))+g(t, x(t), z(t)) \\
\dot{z}(t)= & u(t-h) \\
\dot{a}(t)= & -q a(t)+\frac{q}{1-e^{-q \tau}} u_{\mathrm{nom}}(t, x(t)) \\
& -\frac{q e^{-q \tau}}{1-e^{-q \tau}} u_{\mathrm{nom}}(t-\tau, x(t-\tau))
\end{aligned}\right.
$$

with state space $\mathbb{R}^{n+2 p}$, where the control $u$ will be specified by our theorem. In terms of the variable $s(t)=z(t)-a(t-$ $h$ ), we prove:

Theorem 1 Let Assumptions 1-2 hold. Let h, $\epsilon$, and $m$ be any constants such that

$$
h>0, \quad \epsilon \in\left(0, \frac{1}{2 h}\right), \text { and } m>\left(q+\frac{3.2}{\tau}\right) \bar{u} .
$$

Then, with the control

$$
\begin{aligned}
& u(t-h)=-\epsilon \sigma_{p, 1}(s(t-h))- \\
& \sigma_{p, m}\left(q a(t-h)-\frac{q}{1-e^{-q \tau}} u_{\mathrm{nom}}(t-h, x(t-h))\right. \\
& \left.+\frac{q e^{-q \tau}}{1-e^{-q \tau}} u_{\mathrm{nom}}(t-h-\tau, x(t-h-\tau))\right),
\end{aligned}
$$

all solutions of (1) converge to 0 as $t \rightarrow \infty$.

Before turning to the proof of Theorem 1, we point out several ways in which our control (6) differs from existing controls in the literature. First, our control (6) is bounded by $\sqrt{p}(m+\epsilon)$, and therefore provides a time delayed analog of the bounded backstepping controllers from Mazenc and Bowong (2004), which only applied to undelayed systems. Second, there is no bound on the allowable delay $h>0$. This contrasts with the emulation approach in Mazenc et al. (2008), which usually produces a hard bound on the delay. On the other hand, as we show below, it is sometimes useful to introduce a bound on the delay $h$ to ensure that our Assumption 2 is satisfied. Also, while the usual delay compensation results (based on emulation) conclude uniform global asymptotic stability (UGAS), Theorem 1 does not ensure the uniformness of the asymptotic stability. On the other hand, if we strengthen Assumption 2 to requiring input-to-state stability (as defined in Khalil (2002)) of (3) with respect to $d$, then a slight variant of our proof of Theorem 1 shows that (1) in closed loop with (6) satisfies UGAS to 0 . Finally, notice that the lower bound on $m$ in (5) can be made arbitrarily small by enlarging $\tau>0$ and reducing $q$, while maintaining our requirement that $q \tau<1$. However, $\tau$ must also be such that all trajectories of (3) converge to 0 . See Section 5 below for ways to check the convergence properties for solutions of (3).

\section{Proof of Theorem 1}

Using Assumption 1, we can easily prove that the closedloop system given by (4) and (6) is forward complete. Next, we extend $u_{\text {nom }}$ to $[-h, \infty) \times \mathbb{R}^{n}$ by setting $u_{\text {nom }}(\ell, x)=$ $u_{\text {nom }}(0, x)$ for all $\ell \in[-h, 0]$ and all $x \in \mathbb{R}^{n}$. We also define $\Gamma:[0, \infty) \times C_{\text {in }} \rightarrow \mathbb{R}^{p}$ by

$$
\Gamma(t, \phi)=\int_{-\tau}^{0} \frac{q e^{q v}}{1-e^{-q \tau}} u_{\text {nom }}(t+v, \phi(v)) \mathrm{d} v .
$$

Then

$$
\Gamma\left(t, x_{t}\right)=\int_{t-\tau}^{t} \frac{q e^{q(\ell-t)}}{1-e^{-q \tau}} u_{\text {nom }}(\ell, x(\ell)) \mathrm{d} \ell
$$

for all $t \geq 0$. Also,

$$
\begin{aligned}
\left.\frac{d}{d t} \Gamma\left(t, x_{t}\right)\right)= & -q \Gamma\left(t, x_{t}\right)+\frac{q}{1-e^{-q \tau}} u_{\mathrm{nom}}(t, x(t)) \\
& -\frac{q e^{-q \tau}}{1-e^{-q \tau}} u_{\mathrm{nom}}(t-\tau, x(t-\tau))
\end{aligned}
$$

holds for all $t \geq 0$. Comparing the formula for $\dot{a}(t)$ in (4) with (9), it follows that

$$
\frac{d}{d t}\left(a(t)-\Gamma\left(t, x_{t}\right)\right)=-q\left(a(t)-\Gamma\left(t, x_{t}\right)\right)
$$

for all $t \geq 0$, so since $q$ is a positive constant, $a(t)-\Gamma\left(t, x_{t}\right)$ converges exponentially to zero. Also, our choice $s(t)=$ $z(t)-a(t-h)$ gives

$$
\left\{\begin{aligned}
\dot{x}(t)= & f(t, x(t))+g(t, x(t), s(t)+a(t-h)) \\
\dot{s}(t)= & u(t-h)+q a(t-h) \\
& -\frac{q}{1-e^{-q \tau}} u_{\mathrm{nom}}(t-h, x(t-h)) \\
& +\frac{q e^{-q \tau}}{1-e^{-q \tau}} u_{\mathrm{nom}}(t-h-\tau, x(t-h-\tau))
\end{aligned}\right.
$$

for all $t \geq \tau$. Since $u_{\text {nom }}$ is bounded by $\bar{u}$, and since

$$
\int_{t-\tau}^{t} \frac{q e^{q(\ell-t)}}{1-e^{-q \tau}} \mathrm{d} \ell=1
$$

holds for all $t \geq 0$, we deduce that $\Gamma$ is also bounded by $\bar{u}$. Also,

$$
\frac{\ell}{1-e^{-\ell}} \leq \frac{8}{5} \text { for all } \ell \in(0,1]
$$

because the left side of $(12)$ is increasing in $\ell$ on $(0,1]$.

Since $a(t)-\Gamma\left(t, x_{t}\right)$ exponentially converges to zero and $q \tau<1$, it therefore follows from (6), (12) (with the choice $\ell=q \tau)$, and our lower bound on $m$ from (5) that for each initial function, we have

$$
\begin{aligned}
& u(t-h)=-\epsilon \sigma_{p, 1}(s(t-h)) \\
& -\left\{q a(t-h)-\frac{q}{1-e^{-q \tau}} u_{\mathrm{nom}}(t-h, x(t-h))\right. \\
& \left.+\frac{q e^{-q \tau}}{1-e^{-q \tau}} u_{\mathrm{nom}}(t-h-\tau, x(t-h-\tau))\right\}
\end{aligned}
$$

when $t>0$ is sufficiently large, since each component of the quantity in curly braces in (13) will be valued in $[-m, m]$ when $t$ is large enough, so the saturation $\sigma_{p, m}$ in our control (6) can be omitted. Hence,

$$
\dot{s}(t)=-\epsilon \sigma_{p, 1}(s(t-h))
$$

also holds when $t>0$ is sufficiently large. Also, our bound on $\epsilon>0$ from (5) implies that (14) is globally asymptotically and uniformly locally exponentially stable to 0 ; see the appendix below. Since $a(t)-\Gamma\left(t, x_{t}\right)$ converges exponentially to zero, the $x$ subsystem of $(10)$ is $\dot{x}(t)=$ $f(t, x(t))+g\left(t, x(t), \Gamma\left(t-h, x_{t-h}\right)+d(t)\right)$, where $d(t)=$ $s(t)+a(t-h)-\Gamma\left(t-h, x_{t-h}\right)$ exponentially converges to 0 . Hence, the result follows from Assumption 2, since

$z(t)=s(t)+\left(a(t-h)-\Gamma\left(t-h, x_{t-h}\right)\right)+\Gamma\left(t-h, x_{t-h}\right)$ is a sum of three terms that converge to 0 .

Remark 1 The variable $a(t)$ in (4) makes it possible to 
avoid having the distributed term $\Gamma\left(t, x_{t}\right)$ in the control (6). We can easily extend Theorem 1 to cover

$$
\left\{\begin{array}{l}
\dot{x}(t)=f(t, x(t))+g(t, x(t), z(t)) \\
\dot{z}(t)=-b z(t)+u(t-h)
\end{array}\right.
$$

for any constant $b>0$. Indeed, our choice of a $(t)$ from (4) and the choice $s(t)=z(t)-a(t-h)$ give

$$
\begin{aligned}
\dot{s}(t)= & -b s(t)+u(t-h)+(q-b) a(t-h) \\
& -\frac{q}{1-e^{-q \tau}} u_{\text {nom }}(t-h, x(t-h)) \\
& +\frac{q e^{-q \tau}}{1-e^{-q \tau}} u_{\text {nom }}(t-\tau-h, x(t-h-\tau)) .
\end{aligned}
$$

Hence, we can simply choose

$$
\begin{aligned}
& u(t-h)=-\sigma_{p, m}((q-b) a(t-h) \\
& -\frac{q}{1-e^{-q \tau}} u_{\text {nom }}(t-h, x(t-h)) \\
& \left.+\frac{q e^{-q \tau}}{1-e^{-q \tau}} u_{\text {nom }}(t-\tau-h, x(t-h-\tau))\right)
\end{aligned}
$$

to realize our goal for any constant $m>\left(|q-b|+\frac{3.2}{\tau}\right) \bar{u}$, by a slight variant of our proof of Theorem 1.

\section{Sufficient Conditions for Assumption 2 to Hold}

A key feature of our work is that we do not require the nominal control $u_{\text {nom }}$ to render

$$
\dot{\xi}(t)=f(t, \xi(t))+g\left(t, \xi(t), u_{\text {nom }}(t, \xi(t))\right)
$$

UGAS to 0 . On the other hand, if (18) is control affine (meaning, there is a function $G$ such that $g(t, \xi, u)=$ $G(t, \xi) u$ for all $t \geq 0, \xi \in \mathbb{R}^{n}$, and $u \in \mathbb{R}^{p}$ ) and $u_{\text {nom }}$ is independent of time, then we can give sufficient Lyapunovlike conditions for Assumption 2 to hold, in terms of (18). One way to do so is by using the following, where $V_{t}$ and $V_{\xi}$ denote the partial derivative with respect to $t$ and the gradient with respect to $\xi$, respectively:

Assumption 3 The functions $f:[0, \infty) \times \mathbb{R}^{n} \rightarrow \mathbb{R}^{n}, G$ : $[0, \infty) \times \mathbb{R}^{n} \rightarrow \mathbb{R}^{n \times p}$, and $u_{\text {nom }}: \mathbb{R}^{n} \rightarrow \mathbb{R}^{p}$ are continuous, and $f$ and $G$ are uniformly locally Lipschitz in the second variable. Also, there are a $C^{1}$ uniformly proper and positive definite function $V:[0, \infty) \times \mathbb{R}^{n} \rightarrow[0, \infty)$; a uniformly continuous positive definite function $W: \mathbb{R}^{n} \rightarrow[0, \infty)$; positive constants $\kappa, r_{1}$, and $r_{3}$; and a constant $r_{2} \geq 0$ such that

$$
\begin{aligned}
& V_{t}(t, \xi)+V_{\xi}(t, \xi)\left(f(t, \xi)+G(t, \xi) u_{\text {nom }}(\xi)\right) \leq-W(\xi), \\
& \left|V_{\xi}(t, \xi) G(t, \xi)\right| \leq \kappa \sqrt{W(\xi)},\left|u_{\text {nom }}(\xi)\right| \leq r_{1} \sqrt{W(\xi)}, \\
& |f(t, \xi)| \leq r_{2} \sqrt{W(\xi)}, \text { and }|G(t, \xi)| \leq r_{3}
\end{aligned}
$$

hold for all $\xi \in \mathbb{R}^{n}$ and $t \geq 0$. Also, $u_{\text {nom }}(0)=0$, and $u_{\text {nom }}$ admits a global Lipschitz constant $C>0$.

While the control affine system $\dot{\xi}=f(t, \xi)+G(t, \xi) u_{\text {nom }}(t, \xi)$ in Assumption 3 is not the most general class of nonlinear systems, it is a standard form that occurs in numerous applications, e.g., when one linearizes in the input. Assumption 3 is satisfied by a large class of linear time-varying systems in closed loop with linear feedback controls. More generally, if we are able to choose a control $u_{\text {nom }}$ such that $\dot{\xi}=f(t, \xi)+G(t, \xi) u_{\text {nom }}(t, \xi)$ is globally exponentially stable to 0 and if $G$ is bounded, then (under appropriate Lipschitzness conditions on $f, G$, and $u_{\text {nom }}$ ) (Khalil, 2002, Theorem 4.14) constructs a function $V$ such that Assumption 3 holds with $W(\xi)=|\xi|^{2}$. We prove the following, which is of independent interest (but see Remarks 2-3 for extensions, including a scaling that allows $h$ to be arbitrarily large when the drift $f$ in (18) is the zero function):

Proposition 1 If Assumption 3 holds, and if $h>0$ and $\tau>0$ are any constants such that

$$
h+\tau<\frac{1}{2 C \kappa \sqrt{r_{2}^{2}+2 r_{1}^{2} r_{3}^{2}}},
$$

then there is a constant $q \in(0,1 / \tau)$ such that for each continuous function $d \in \mathcal{L}^{2}(p,[0, \infty))$, the following holds: All solutions of

$$
\begin{aligned}
& \dot{\xi}(t)=f(t, \xi(t))+ \\
& G(t, \xi(t))\left(\int_{t-\tau-h}^{t-h} \frac{q e^{q(\ell-t+h)}}{1-e^{-q \tau}} u_{\text {nom }}(\xi(\ell)) \mathrm{d} \ell+d(t)\right)
\end{aligned}
$$

converge to 0 as $t \rightarrow \infty$. If, in addition, $u_{\text {nom }}$ is bounded, then Assumption 2 holds with $g(t, \xi, u)=G(t, \xi) u$.

Proof: Choose any function $d$ as in the statement of the proposition and any solution $\xi(t)$ of (21). In the rest of the proof, we only consider values of $t \geq \tau+h$. Then our bounds in (19), our Lipschitz constant $C$ for $u_{\text {nom }}$, and (11) imply that the time derivative $\dot{V}$ of $V$ from (19), along all trajectories of (21), satisfies

$$
\begin{aligned}
\dot{V} \leq & -W(\xi(t))+V_{\xi}(t, \xi(t)) G(t, \xi(t))(d(t)+ \\
& \left.\int_{t-\tau}^{t} \frac{q e^{q(\ell-t)}}{1-e^{-q \tau}}\left(u_{\mathrm{nom}}(\xi(\ell-h))-u_{\mathrm{nom}}(\xi(t))\right) \mathrm{d} \ell\right) \\
\leq & -W(\xi(t))+\kappa \sqrt{W(\xi(t))}(d(t) \\
& \left.C \int_{t-\tau}^{t} \frac{q e^{q(\ell-t)}}{1-e^{-q \tau}}|\xi(\ell-h)-\xi(t)| \mathrm{d} \ell\right) \\
\leq & -W(\xi(t)) \\
& +\kappa \sqrt{W(\xi(t))}\left(C \int_{t-\tau-h}^{t}|\dot{\xi}(\ell)| \mathrm{d} \ell+|d(t)|\right),
\end{aligned}
$$

where $q$ will be specified and the last inequality uses (11) and the fact that

$$
|\xi(\ell-h)-\xi(t)| \leq \int_{t-h-\tau}^{t}|\dot{\xi}(p)| \mathrm{d} p
$$

for all $\ell \in[t-\tau, t]$. Hence, Jensen's inequality gives

$$
\begin{aligned}
\dot{V} \leq & -\frac{1}{2} W(\xi(t))+(\tau+h) \kappa^{2} C^{2} \int_{t-\tau-h}^{t}|\dot{\xi}(\ell)|^{2} \mathrm{~d} \ell \\
& +\kappa^{2}|d(t)|^{2},
\end{aligned}
$$

where we also used Young's inequality to get $b \sqrt{W(\xi(t))} \leq$ $0.25 W(\xi(t))+b^{2}$ twice for suitable $b \geq 0$. Also, Assumption $3,(21)$, two applications of the triangle inequality, and Jensen's inequality give

$$
\begin{aligned}
|\dot{\xi}(t)|^{2} \leq & 2|f(t, \xi(t))|^{2}+2|G(t, \xi(t))|^{2}\left(5|d(t)|^{2}\right. \\
& \left.+\frac{5}{4}\left|\int_{t-\tau}^{t} \frac{q e^{q(\ell-t)}}{1-e^{-q \tau}} u_{\text {nom }}(\xi(\ell-h)) \mathrm{d} \ell\right|^{2}\right) \\
\leq & 2 r_{2}^{2} W(\xi(t))+10 r_{3}^{2}|d(t)|^{2} \\
& +\frac{5}{2} r_{3}^{2} r_{1}^{2} \tau \int_{t-\tau}^{t} \frac{q^{2} e^{2 q(\ell-t)}}{\left(1-e^{-q \tau}\right)^{2}} W(\xi(\ell-h)) \mathrm{d} \ell,
\end{aligned}
$$


since $(a+b)^{2} \leq(5 / 4) a^{2}+5 b^{2}$ holds for all $a \geq 0$ and $b \geq 0$. Set

$$
\begin{aligned}
& V_{1}(t)= \\
& V(\xi(t))+(\tau+h) \kappa^{2} C^{2} \int_{t-\tau-h}^{t} \int_{m}^{t}|\dot{\xi}(\ell)|^{2} \mathrm{~d} \ell \mathrm{d} m
\end{aligned}
$$

and $\bar{\Delta}=\kappa^{2}\left(10(\tau+h)^{2}\left(C r_{3}\right)^{2}+1\right)$. Combining (23)-(24) and setting $\ell=q \tau \in(0,1)$ in (12), we get

$$
\begin{aligned}
\dot{V}_{1} \leq & -\frac{1}{2} W(\xi(t))+(\tau+h)^{2} \kappa^{2} C^{2}|\dot{\xi}(t)|^{2}+\kappa^{2}|d(t)|^{2} \\
\leq & \left(-\frac{1}{2}+2(\tau+h)^{2} \kappa^{2} C^{2} r_{2}^{2}\right) W(\xi(t))+\bar{\Delta}|d(t)|^{2} \\
& +\frac{4\left((\tau+h) \kappa C r_{1} r_{3}\right)^{2} q}{1-e^{-q \tau}} \int_{t-\tau}^{t} W(\xi(\ell-h)) \mathrm{d} \ell .
\end{aligned}
$$

Hence, the time derivative of

$$
\begin{aligned}
& V_{2}(t)=V_{1}(t)+\frac{4\left((\tau+h) \kappa C r_{1} r_{3}\right)^{2} q}{1-e^{-q \tau}}\left(\tau \int_{t-h}^{t} W(\xi(\ell)) \mathrm{d} \ell\right. \\
& \left.+\int_{t-\tau-h}^{t-h} \int_{m}^{t-h} W(\xi(\ell)) \mathrm{d} \ell \mathrm{d} m\right)
\end{aligned}
$$

along all trajectories of (21) satisfies

$$
\begin{aligned}
& \dot{V}_{2} \leq\left\{-\frac{1}{2}+2\left((\tau+h) \kappa C r_{2}\right)^{2}\right. \\
& \left.+\frac{4\left((\tau+h) \kappa C r_{1} r_{3}\right)^{2} q \tau}{1-e^{-q \tau}}\right\} W(\xi(t))+\bar{\Delta}|d(t)|^{2} .
\end{aligned}
$$

Since $\lim _{q \rightarrow 0^{+}} q \tau /\left(1-e^{-q \tau}\right)=1$ for each $\tau>0$, it follows from our bound (20) that for small enough $q \in(0,1 / \tau)$, the quantity in curly braces in (28) is a negative constant. Therefore, since $d \in \mathcal{L}^{2}(p,[0, \infty))$, we can integrate (28) on $[h+\tau, t]$ for any $t \geq \tau+h$ to conclude that $V_{2}(t)$ is bounded, so since $V$ is uniformly proper and positive definite, it follows that $|\xi(t)|$ is bounded. Hence, $W(\xi(t))$ is a uniformly continuous function of $t$, and (28) gives $\int_{\tau+h}^{\infty} W(\xi(\ell)) \mathrm{d} \ell<\infty$. It follows from Barbalat's Lemma that $\lim _{t \rightarrow \infty} \xi(t)=0$, as needed.

Remark 2 While stated as an existence result for a constant $q \in(0,1 / \tau)$, the proof of Proposition 1 can be used to find an explicit bound on the allowable values of $q$, namely, $q$ must be chosen such that the quantity in curly braces in (28) is negative. Reducing $q>0$ may reduce the convergence rate for the closed loop system given by (4) and (6), since $\frac{d}{d t}\left(a(t)-\Gamma\left(t, x_{t}\right)\right)=-q\left(a(t)-\Gamma\left(t, x_{t}\right)\right)$ for all $t$. On the other hand, we can use (12) with the choice $\ell=q \tau \in(0,1)$ to show that for all $q \in(0,1 / \tau)$, the quantity in curly braces in (28) is bounded above by $-0.5+2\left((\tau+h) \kappa C r_{2}\right)^{2}+6.4((\tau+$ h) $\left.\kappa C r_{1} r_{3}\right)^{2}$, and this upper bound is negative if

$$
h+\tau<\frac{1}{2 C \kappa \sqrt{r_{2}^{2}+3.2 r_{1}^{2} r_{3}^{2}}} .
$$

Hence, our proof of Proposition 1 leads to the following variant of Proposition 1 that applies for any $q \in(0,1 / \tau)$ : If Assumption 3 holds, and if $h>0$ and $\tau>0$ are any constants such that (29) holds, then for all $q \in(0,1 / \tau)$ and each continuous function $d \in \mathcal{L}^{2}([0, \infty))$, the following holds: All solutions of (21) converge to 0 as $t \rightarrow \infty$. If, in addition, $u_{\text {nom }}$ is bounded, then Assumption 2 holds with $g(t, \xi, u)=G(t, \xi) u$.

Remark 3 Consider the special case of Assumption 3 where $V$ is independent of $t$, and where the drift term $f(t, \xi)$ in (18) is the zero function. Then, we can choose $r_{2}=0$ in Assumption 3. Also, for any constant $\eta \in(0,1)$, Assumption 3 still holds if we replace $u_{\text {nom }}, W, C, r_{1}$, and $\kappa$ by $\eta u_{\text {nom }}, \eta W, \eta C, \sqrt{\eta} r_{1}$, and $\kappa / \sqrt{\eta}$, respectively in (19). With these replacements, our upper bound (20) changes to

$$
h+\tau<\frac{1}{2 \eta C \kappa \sqrt{2 r_{1}^{2} r_{3}^{2}}} \rightarrow \infty
$$

as $\eta \rightarrow 0$. Hence, Proposition 1 applies for any $h>0$, by scaling the nominal control $u_{\text {nom }}$ by a small enough constant $\eta>0$. Then, Theorem 1 implies asymptotic convergence of all trajectories of (1) to 0 (with the control affine choice $g(t, \xi, u)=G(t, \xi) u)$ for all $h>0$ when we use the control (6) and the nominal control $\eta u_{\text {nom }}$. See Section 7 for an extension of this scaling argument that applies when $V$ is not time invariant.

\section{First Example: Output Feedback}

Consider the two-dimensional system

$$
\dot{x}(t)=\sigma_{1}(|x(t)|)+z(t), \quad \dot{z}(t)=u(t-h)
$$

for any delay $h>0$, with the choice

$$
u_{\mathrm{nom}}(x)=-2 \sigma_{1}(\max \{0, x\}) .
$$

Then $\sigma_{1}(|\xi|)+u_{\text {nom }}(\xi)=-\sigma_{1}(\xi)$ for all $\xi \in \mathbb{R}$, so Assumption 3 holds with the choices

$$
V(\xi)=\int_{0}^{\xi} \sigma_{1}(r) \mathrm{d} r
$$

$f(t, \xi)=\sigma_{1}(|\xi|), G(t, \xi)=1, W(\xi)=\sigma_{1}^{2}(\xi), C=r_{1}=2$, and $r_{2}=r_{3}=\kappa=1$. Therefore, since $\tau$ in Proposition 1 can be taken as small as desired, this leads to the bound

$$
h<\frac{1}{2 C \kappa \sqrt{r_{2}^{2}+2 r_{1}^{2} r_{3}^{2}}} \approx 0.1 .
$$

This restriction on $h$ is not surprising, because the local exponential instability of $\dot{x}(t)=\sigma_{1}(|x(t)|)$ strongly suggests that (31) cannot be stabilized by a feedback that does not incorporate past values of itself (as feedbacks provided by the reduction model approach do) when $h$ is larger than a certain value. In the next section, we give an example that allows arbitrarily long delays.

One advantage of the feedback from Theorem 1 is that it does not depend on negative values of $x$. Hence, if only the output $y=(\max \{0, x\}, z)$ is measured, then our result still applies. To the best of our knowledge, no other result in the literature can be applied to this system when only $y=(\max \{0, x\}, z)$ is measured. One cannot apply classical backstepping if no negative values of $x$ can be measured. Classical backstepping would entail finding a $C^{2}$ fictitious feedback $z_{f}(x)$ such that $\dot{x}=\sigma_{1}(|x|)+z_{f}(x)$ is globally asymptotically stable to 0 . Since $z_{f}$ is $C^{2}$ and $z_{f}(0)=0$, it admits a decomposition of the form $z_{f}(x)=a x+b(x) x^{2}$ in some interval around 0 , for some constant $a \in \mathbb{R}$. Therefore, when $x>0$ is small enough, we get $\dot{x}=\sigma_{1}(|x|)+z_{f}(x)=$ $x+a x+b(x) x^{2}$. We deduce that $a \leq-1$ must hold, because we need $\dot{x} \leq 0$ when $x>0$ is close to 0 . If $z_{f}(x)$ only depends on nonnegative values of $x$, then $a x+b(x) x^{2}$ is constant over $(-\infty, 0]$, which is impossible because $a \leq$ 
-1 . This is perhaps one of the most important advantages of our new approach, namely, if $u_{\text {nom }}$ only depends on an output $y$, then we only need that output.

Although our approach has important advantages, Theorem 1 is an asymptotic convergence result that does not provide information about transient behavior of the system with our control (6), and so differs from results in the literature that prove uniform global asymptotic stability. Therefore, we next provide simulations of the closed loop dynamics, to show the transient behavior. In our simulations, we took the delay $h=0.09, \tau=1, q=0.5$, $\epsilon=1 /(4 h)=25 / 9$, and $m=2.1(q+3.2 / \tau)=7.77$, which satisfy our requirements. We took the constant initial function $(1,1)$ for the dynamics. In Figure 1 below, we show the simulations we obtained using Mathematica; see Mathematica (2015). While our simulations show transient movement of the state away from the equilibrium, they show asymptotic convergence to the equilibrium and therefore help illustrate the value of Theorem 1 .

\section{Second Example: Arbitrarily Long Delays}

In the preceding example, we imposed an upper bound on the allowable delay $h$. In this section, we provide an application of Theorem 1 that allows the delay to be arbitrarily large. Consider the system

$$
\left\{\dot{x}(t)=\cos ^{2}(t) z(t), \quad \dot{z}(t)=u(t-h) .\right.
$$

We verify Assumption 3 with $u_{\text {nom }}(\xi)=-\eta \sigma_{1}(\xi)$, where the constant $\eta \in(0,1]$ will be chosen later. We show that Assumption 3 is satisfied with the choices

$$
\begin{aligned}
& V(t, \xi)=V_{0}(\xi)+\frac{\eta}{\pi} \frac{V_{0}(\xi)}{1+V_{0}(\xi)} \int_{t-\pi}^{t} \int_{s}^{t} \cos ^{2}(r) \mathrm{d} r \mathrm{~d} s \\
& \text { where } V_{0}(\xi)=\int_{0}^{\xi} \sigma_{1}(p) \mathrm{d} p, f(t, \xi)=0, r_{3}=1, \\
& G(t, \xi)=\cos ^{2}(t), W(\xi)=\frac{\eta}{2} \frac{V_{0}(\xi)}{1+V_{0}(\xi)}, r_{2}=0 \\
& \kappa=(1+\eta \pi / 2) \sqrt{6 / \eta}, r_{1}=\sqrt{6 \eta}, \text { and } C=\eta .
\end{aligned}
$$

Note that $V_{0}$ is $C^{1}$, even, and (uniformly) proper and positive definite. Also,

$$
\dot{\xi}=f(t, \xi)+G(t, \xi) u_{\text {nom }}(\xi)=-\eta \cos ^{2}(t) \sigma_{1}(\xi) .
$$

Hence, along all trajectories of $\dot{\xi}=f(t, \xi)+G(t, \xi) u_{\text {nom }}(\xi)$, we have $\dot{V}_{0}(\xi)=-\eta \cos ^{2}(t) \sigma_{1}^{2}(\xi)$, and so also

$$
\begin{aligned}
& V_{t}(t, \xi)+V_{\xi}(t, \xi)\left[f(t, \xi)+G(t, \xi) u_{\text {nom }}(\xi)\right] \\
& =-\eta \sigma_{1}^{2}(\xi) \cos ^{2}(t)\left[1+\frac{\eta}{\pi} \frac{1}{\left(1+V_{0}(\xi)\right)^{2}} \int_{t-\pi}^{t} \int_{s}^{t} \cos ^{2}(r) \mathrm{d} r \mathrm{~d} s\right] \\
& +\frac{\eta}{\pi} \frac{V_{0}(\xi)}{1+V_{0}(\xi)}\left[\pi \cos ^{2}(t)-\int_{t-\pi}^{t} \cos ^{2}(r) \mathrm{d} r\right] \\
& \leq-\frac{\eta}{\pi} \frac{V_{0}(\xi)}{1+V_{0}(\xi)} \int_{t-\pi}^{t} \cos ^{2}(r) \mathrm{d} r=-W(\xi),
\end{aligned}
$$

since $V_{0}(\xi)=\frac{1}{2} \xi^{2}$ for all $\xi \in[-1,1]$, which gives $V_{0}(\xi) /(1+$ $\left.V_{0}(\xi)\right) \leq \sigma_{1}^{2}(\xi)$ for all $\xi \in \mathbb{R}$. Also,

$$
\begin{aligned}
& \left|V_{\xi}(t, \xi)\right|= \\
& \left|\sigma_{1}(\xi)+\frac{\eta}{\pi} \frac{\sigma_{1}(\xi)}{\left(1+V_{0}(\xi)\right)^{2}} \int_{t-\pi}^{t} \int_{s}^{t} \cos ^{2}(r) \mathrm{d} r \mathrm{~d} s\right| \\
& \leq(1+\eta \pi / 2)\left|\sigma_{1}(\xi)\right|
\end{aligned}
$$
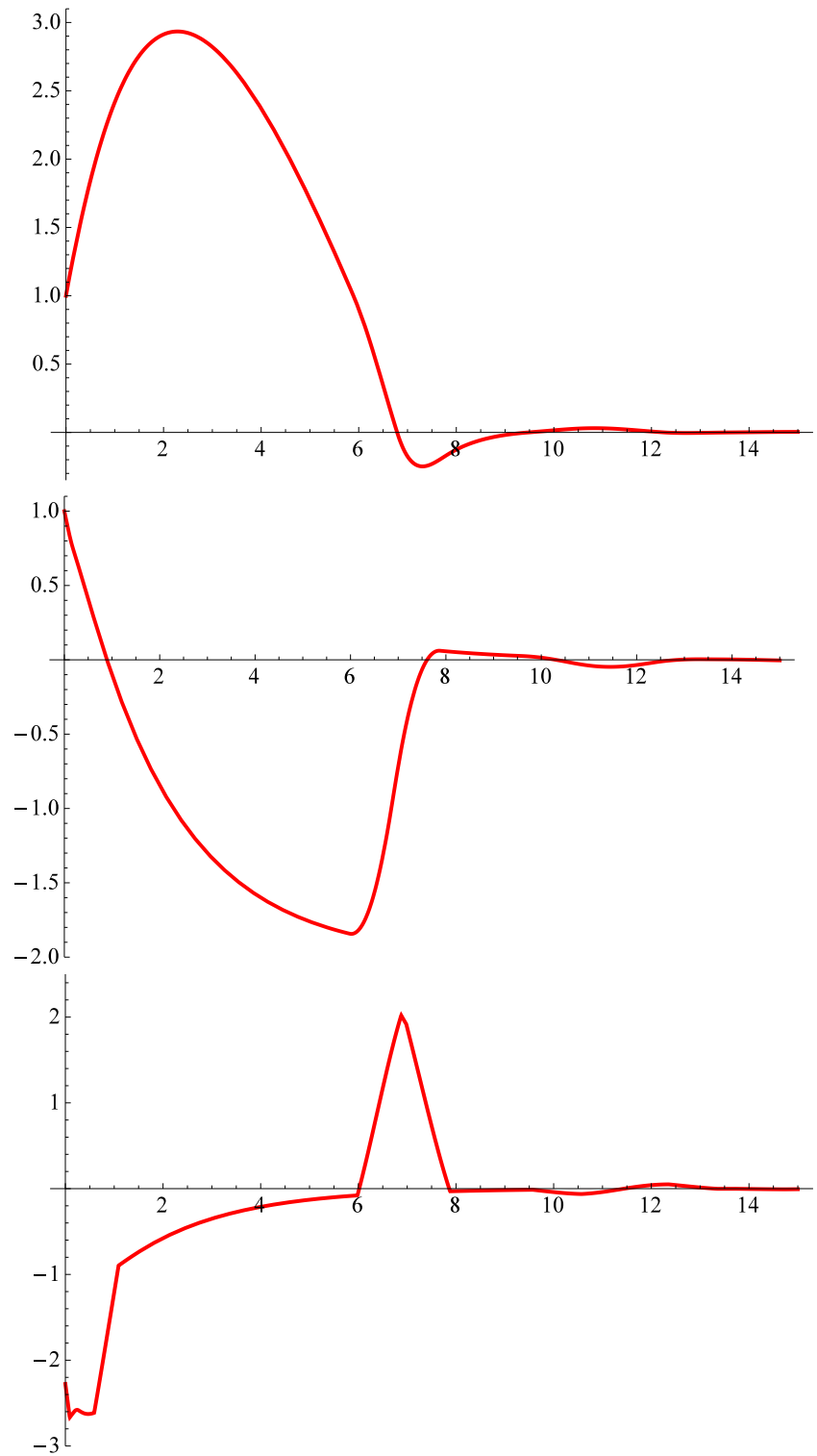

Fig. 1. Closed Loop System Performance of System (31) from First Example with Stabilizing Delay Compensating Control (6). Trajectory for $x$ (Top Panel), $z$ (Middle Panel), and Control $u$ (Bottom Panel).

for all $t \geq 0$ and $\xi \in \mathbb{R}$. If $|\xi| \geq 1$, then since $V_{0}$ is nondecreasing on $[0, \infty)$ and even, we get

$$
\begin{aligned}
& \kappa \sqrt{W(\xi)} \geq \kappa \sqrt{\frac{\eta V_{0}(1)}{2\left(1+V_{0}(1)\right)}}=\kappa \sqrt{\frac{0.5 \eta}{2(1+0.5)}}= \\
& \frac{\kappa \sqrt{\eta}}{\sqrt{6}}=(1+\eta \pi / 2)\left|\sigma_{1}(\xi)\right| \geq\left|V_{\xi}(t, \xi) G(t, \xi)\right| .
\end{aligned}
$$

On the other hand, if $|\xi|<1$, then since $V_{0}(\xi)=\frac{1}{2} \xi^{2} \leq \frac{1}{2}$, we get

$$
\begin{aligned}
& \kappa \sqrt{W(\xi)} \geq \frac{\kappa}{\sqrt{2}} \sqrt{\frac{\eta \xi^{2}(t) / 2}{3 / 2}}=\frac{\kappa \sqrt{\eta}}{\sqrt{6}}\left|\sigma_{1}(\xi)\right| \\
& =(1+\eta \pi / 2)\left|\sigma_{1}(\xi)\right| \geq\left|V_{\xi}(t, \xi) G(t, \xi)\right| .
\end{aligned}
$$

This also gives $\left|u_{\text {nom }}(\xi)\right| \leq r_{1} \sqrt{W(\xi)}$, and the remaining requirements from Assumption 3 are satisfied because $f \equiv$ 
0 and $G$ is bounded by 1 . Also, with the choices (35), our upper bound (20) is $\tau+h<1 /(16.97 \eta(1+\eta \pi / 2))$, so we can allow any constant delay $h>0$ as long as $\eta>0$ satisfies

$$
\eta(1+\eta \pi / 2)<\frac{1}{16.97(h+\tau)} .
$$

Notice that this example used a scaling of the control to compensate arbitrarily long delays, even though $V$ is timevarying. Therefore, this example provides an extension of the scaling argument from Remark 3.

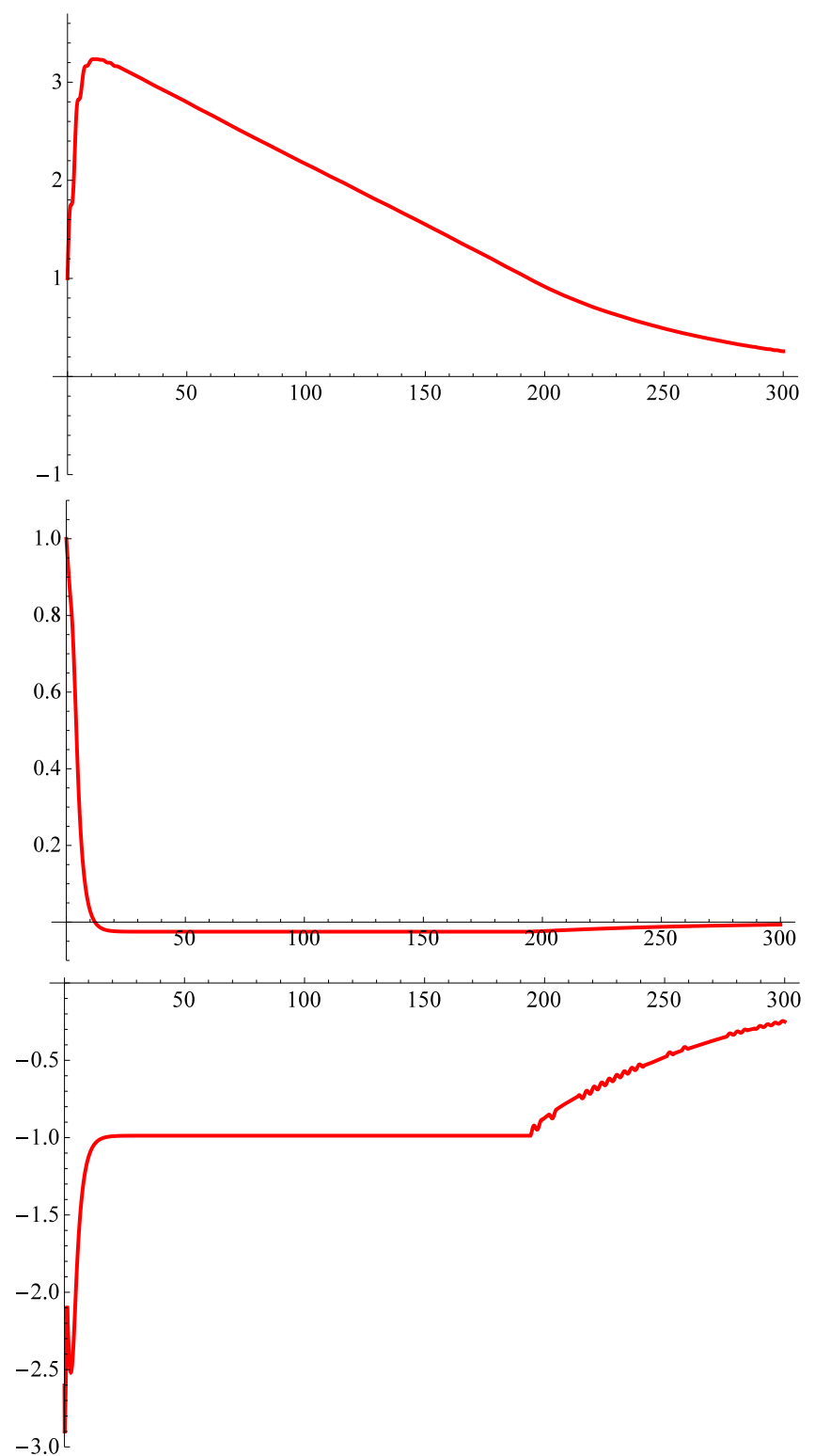

Fig. 2. Closed Loop System Performance of System (34) from Second Example with Stabilizing Delay Compensating Control (6). Trajectory for $x$ (Top Panel), $z$ (Middle Panel), and Control $u$ (Bottom Panel).

Figure 2 illustrates the performance of (34) with our bounded delay compensating controller (6). The figure shows plots from a Mathematica simulation of the con- trol loop system with the same constant initial function $(1,1)$ that we used to simulate $(31)$. We also used the delay $h=1, q=0.5, \tau=1, \epsilon=1 /(4 h)=0.25$, the scaling parameter $\eta=0.025$, and the saturation bound $m=(q+3.2 / \tau) \eta=0.0925$ from our requirements. With the longer delay of $h=1$, the convergence towards the zero equilibrium is slower than in the Section 6 . However, our simulation illustrates the transient behavior in the $x$ variable and convergence toward the equilibrium, and therefore helps illustrate our theorem.

\section{Comparison with Earlier Backstepping Results}

As we noted above, some potential benefits of our approach are that we can ensure boundedness of the control for the overall cascade (4), that the nominal control does not need to be differentiable, and that no Lie derivatives of Lyapunov functions are needed in the control design. This contrasts with our earlier work Mazenc et al. (2008), which produced globally asymptotically stabilizing controllers for systems of the form

$$
\begin{aligned}
& \dot{x}(t)=f(x(t))+G(x(t)) z(t) \\
& \dot{z}(t)=u(x(t-2 h), x(t-h), z(t-h)),
\end{aligned}
$$

with state space $\mathbb{R}^{n} \times \mathbb{R}^{m}$, under the following assumption:

Assumption 4 The functions $f: \mathbb{R}^{n} \rightarrow \mathbb{R}^{n}$ and $G: \mathbb{R}^{n} \rightarrow$ $\mathbb{R}^{n \times m}$ are locally Lipschitz. Also, there are a $C^{1}$ function $u_{s}: \mathbb{R}^{n} \rightarrow \mathbb{R}^{m}$ such that $u_{s}(0)=0$; a function $\gamma \in \mathcal{K}_{\infty}$ for which $\gamma(r) \leq r$ for all $r \geq 0$; a $C^{1}$ uniformly proper and positive definite function $V: \mathbb{R}^{n} \rightarrow[0, \infty)$; positive constants $\bar{L}$ and $K_{1}$; and constants $K_{i} \geq 0(i=2,3,4)$ such that

$$
\begin{aligned}
& \nabla V(x)\left[f(x)+G(x) u_{s}(x)\right] \leq-\gamma^{2}(\sqrt{n}|x|) \\
& |\nabla V(x) G(x)| \leq K_{1} \gamma(|x|), \quad\left|\nabla u_{s}(x)\right| \leq \bar{L} \\
& |f(x)|^{2} \leq K_{2} \gamma^{2}(|x|), \quad|G(x)|^{2} \leq K_{3}(\gamma(|x|)+1) \\
& \text { and }\left[|G(x)|\left|u_{s}(q)\right|\right]^{2} \leq K_{4}\left[\gamma^{2}(|x|)+\gamma^{2}(|q|)\right]
\end{aligned}
$$

hold for all $x \in \mathbb{R}^{n}$ and $q \in \mathbb{R}^{n}$.

Then Mazenc et al. (2008) proves that for any constant delay $h>0$ satisfying

$$
h<\min \left\{\frac{1}{\sqrt{8}}, \frac{0.49}{\bar{L} K_{1} \sqrt{2 K_{2}+8 K_{4}+0.25}}\right\},
$$

the dynamics (40) in closed loop with

$$
\begin{aligned}
& u(x(t-2 h), x(t-h), z(t-h))= \\
& -z(t-h)+u_{s}(x(t-2 h)) \\
& +\frac{\partial u_{s}}{\partial x}(x(t-h))(f(x(t-h))+G(x(t-h)) z(t-h))
\end{aligned}
$$

satisfies UGAS to zero. Also, Mazenc et al. (2008) shows input-to-state stability with respect to additive uncertainty on the control $u$ in (40). However, (43) will not be bounded in general, even if the nominal control $u_{s}$ is bounded, and Mazenc et al. (2008) requires the $C^{1}$ property for $u_{s}$. Therefore, our current work is a potential improvement over the backstepping results (from Mazenc and Bliman (2006); 
Mazenc et al. (2008)) and other results that do not ensure boundedness of the control, or which require more regularity (such as $C^{1}$ ) for the nominal control.

\section{Conclusions}

Time delays arise in many applications, and often as input delays. Two approaches to stabilization under input delays involve (a) solving the stabilization problem with the delay set to zero and then finding upper bounds on the constant delays $h>0$ that can be introduced into the controller such that the input delayed system with delay $h$ is still asymptotically stable and (b) predictive methods that use dynamic controllers to compensate arbitrarily long delays. In both approaches, backstepping is important. Backstepping involves recursively building controllers, using nominal controllers for subsystems. When input constraints and delays are both present, backstepping can be challenging. While Mazenc and Bowong (2004) provided a bounded backstepping approach for systems without delays, here we extended Mazenc and Bowong (2004) to systems with bounded controls and input delays.

Other potential advantages of our new method are that (a) there are no distributed terms in our control of the type that would arise from prediction and (b) we allow nominal controllers that are not necessarily $C^{1}$. Moreover, there are interesting cases where our controllers apply without imposing any upper bound on the allowable delays. We hope to extend our work to cover cases where there are both input constraints and state constraints or where the system is governed by a hyperbolic partial differential equation, either with a boundary control or with a control in the PDE.

\section{Appendix}

We prove the global asymptotic and uniform local exponential stability of the system (14) for all constants

$$
\epsilon \in\left(0, \frac{1}{2 h}\right)
$$

which was needed in our proof of Theorem 1. Fix any $i \in$ $\{1,2, \ldots, p\}$. Next, note that $\left|\sigma_{1}\left(s_{i}(t)\right)-\sigma_{1}\left(s_{i}(t-h)\right)\right| \leq$ $\left|s_{i}(t)-s_{i}(t-h)\right| \leq h \epsilon$ holds along all trajectories of the system. Therefore, if $s_{i}(t) \geq 1$, then (A.1) gives $\dot{s}_{i}(t) \leq$ $-\epsilon \sigma_{1}\left(s_{i}(t)\right)+h \epsilon^{2}=\epsilon(-1+h \epsilon) \leq-0.5 \epsilon$. On the other hand, if $s_{i}(t) \leq-1$, then $\dot{s}_{i}(t) \geq-\epsilon \sigma_{1}\left(s_{i}(t)\right)-h \epsilon^{2}=$ $\epsilon(1-h \epsilon) \geq 0.5 \epsilon$. This provides a function $T$ such that the following conditions hold for all $i \in\{1,2, \ldots, p\}$ and all initial functions $\phi$ for the dynamics: (i) $\left|s_{i}(t)\right| \leq 1$ for all $t \geq T\left(|\phi|_{\infty}\right)$ and (ii) $T(p)=0$ for all $p \in[0,1]$. This implies that (14) becomes $\dot{s}(t)=-\epsilon s(t-h)$ for all $t \geq T\left(|\phi|_{\infty}\right)+h$, as well as for all $s_{0}$ such that $\left|s_{0}\right|_{\infty} \leq 1$. Therefore, if $t \geq$ $T\left(|\phi|_{\infty}\right)+h$, then we can use Jensen's inequality and the triangle inequality to check that $V_{0}(s)=0.5|s|^{2}$ satisfies

$$
\begin{aligned}
\frac{d}{d t} V_{0}(s(t)) & =-\epsilon|s(t)|^{2}+\epsilon s^{\top}(t)(s(t)-s(t-h)) \\
& \leq-\frac{\epsilon}{2}|s(t)|^{2}+\frac{\epsilon^{3} h}{2} \int_{t-2 h}^{t}|s(r)|^{2} \mathrm{~d} r
\end{aligned}
$$

along the trajectories of (14). Hence, since $\epsilon^{3} h<\frac{\epsilon}{4 h}$, the constant $\ell_{0}=\epsilon /(8 h)-\epsilon^{3} h / 2>0$ is such that

$$
V_{1}(t)=V_{0}(s(t))+\frac{\epsilon}{8 h} \int_{t-2 h}^{t} \int_{\ell}^{t}|s(r)|^{2} \mathrm{~d} r \mathrm{~d} \ell
$$

satisfies

$$
\frac{d}{d t} V_{1}(t) \leq-\frac{\epsilon}{4}|s(t)|^{2}-\ell_{0} \int_{t-2 h}^{t}|s(r)|^{2} \mathrm{~d} r
$$

for all $t \geq T\left(|\phi|_{\infty}\right)+h$. This provides a constant $c_{*}>0$ such that $(d / d t) V_{1}(t) \leq-c_{*} V_{1}(t)$ for all $t \geq T\left(|\phi|_{\infty}\right)+$ $h$, and this gives the uniform global asymptotic and local exponential stability properties, since we also have $\left|s_{i}(t)\right| \leq$ $\left|s_{i}(0)\right| \exp \left(T\left(|\phi|_{\infty}\right)-t\right) e^{h}$ for all $t \in\left[0, T\left(\left|s_{0}\right|_{\infty}\right)+h\right]$ when the $i$-th component $\phi_{i}$ of the initial function $\phi$ satisfies $\left|\phi_{i}\right|_{\infty} \geq 1$.

\section{References}

Bekiaris-Liberis, N. and M. Krstic (2013). Compensation of state-dependent input delay for nonlinear systems. IEEE Transactions on Automatic Control 58(2), 275-289.

Bresch-Pietri, D., J. Chauvin and N. Petit (2012). Adaptive control scheme for uncertain time-delay systems. Automatica 48(8), 1536-1552.

Choi, H.-L. and J-T. Lim (2010). Asymptotic stabilization of an input-delayed chain of integrators with nonlinearity. Systems and Control Letters 59(6), 374-379.

Karafyllis, I. (2002). Non-uniform stabilization of control systems. IMA Journal of Mathematical Control and Information 19(4), 419-444.

Karafyllis, I. and M. Krstic (2014). Numerical schemes for nonlinear predictor feedback. Mathematics of Control, Signals, and Systems 26(4), 519-546.

Karafyllis, I., M. Malisoff, M. de Queiroz, M. Krstic and R. Yang (2015). Predictor-based tracking for neuromuscular electrical stimulation. International Journal of Robust and Nonlinear Control 25(14), 2391-2419.

Khalil, H. (2002). Nonlinear Systems, Third Edition. Prentice Hall. Englewood Cliffs, NJ.

Kharitonov, V. and S-I. Niculescu (2003). On the stability of linear systems with uncertain delay. IEEE Transactions on Automatic Control 48(1), 127-132.

Krstic, M. (2009). Delay Compensation for Nonlinear, Adaptive, and PDE Systems. Systems Control: Foundations and Applications. Birkhauser. Boston.

Krstic, M. and N. Bekiaris-Liberis (2013). Nonlinear Control Under Nonconstant Delays. Society for Industrial and Applied Mathematics. Philadelphia.

Malisoff, M. and F. Mazenc (2009). Constructions of Strict Lyapunov Functions. Communications and Control Engineering Series. Springer. New York.

Mathematica (2015). The world's definitive system for modern technical computing. Available online at: http://www.wolfram.com/mathematica/.

Mazenc, F. and M. Malisoff (2015). Bounded backstepping approach under input delays. In Proceedings of the European Control Conference. Linz, Austria. pp. 2056-2061.

Mazenc, F. and P-A. Bliman (2006). Backstepping design for time-delay nonlinear systems. IEEE Transactions on 
Automatic Control 51(1), 149-154.

Mazenc, F. and S. Bowong (2004). Backstepping with bounded feedbacks for time-varying systems. SIAM Journal on Control and Optimization 43(3), 856-871.

Mazenc, F., M. Malisoff and Z. Lin (2008). Further results on input-to-state stability for nonlinear systems with delayed feedbacks. Automatica 44(9), 2415-2421.

Mazenc, F., S-I. Niculescu and M. Bekaik (2011). Backstepping for nonlinear systems with delay in the input revisited. SIAM Journal on Control and Optimization 49(6), 2263-2278.

Niculescu, S.-I. (2001). Delay Effects on Stability: A Robust Control Approach. Lecture Notes in Control and Information Sciences, Volume 269. Springer. Berlin, Germany.

Smith, O. (1959). A controller to overcome dead time. ISA Journal 6(2), 28-33. 